\title{
A simple endoscopic method with radial endobronchial ultrasonography for low-migration rate coil-tailed fiducial marker placement
}

\author{
Samy Lachkar ${ }^{1}$, Florian Guisier ${ }^{1,2}$, Maxime Roger ${ }^{1}$, Suzanna Bota ${ }^{1}$, Delphine Lerouge ${ }^{3}$, Nicolas Piton ${ }^{4}$, \\ Luc Thiberville ${ }^{1,2}$, Mathieu Salaün ${ }^{1,2}$ \\ ${ }^{1}$ Department of Pulmonology, Thoracic Oncology and Respiratory Intensive Care \& CIC-CRB 1404, Rouen University Hospital, Rouen, France; \\ ${ }^{2}$ QuantIF-LITIS EA 4108, IRIB, Rouen University, Rouen, France; ${ }^{3}$ Department of Oncology radiotherapy, CRLCC F. Baclesse, Caen, France; \\ ${ }^{4}$ Department of Pathology, Rouen University Hospital, Rouen, France \\ Contributions: (I) Conception and design: S Lachkar, F Guisier, L Thiberville, M Salaün; (II) Administrative support: None; (III) Provision of study \\ materials or patients: All authors; (IV) Collection and assembly of data: S Lachkar, F Guisier, D Lerouge; (V) Data analysis and interpretation: S \\ Lachkar, F Guisier, L Thiberville, M Salaun; (VI) Manuscript writing: All authors; (VII) Final approval of manuscript: All authors. \\ Correspondence to: Dr. Samy Lachkar, MD. Department of Pulmonology, Thoracic Oncology and Respiratory Intensive Care, Hôpital Charles \\ Nicolle, CHU de Rouen, 1 rue de Germont, 76031 Rouen Cedex, France. Email: samy.lachkar@chu-rouen.fr.
}

Background: Fiducial markers (FMs) are useful for tracking small peripheral lung nodules (PLN) before stereotactic radiotherapy, but migration over the course of treatment may result in inaccurate dosing to the tumor. To minimize FM migration, coil-tailed FMs have been designed. Our objective was to assess both the feasibility of radial endobronchial ultrasonography (r-EBUS) placement and the migration rate of coil-tailed FMs.

Methods: In this retrospective study, we included patients who received r-EBUS guided placement of coiltailed FMs for PLN <25 mm from June 2015 to May 2018. We introduced the FM into the nodule with the use of bronchial brush, without fluoroscopy.

Results: Thirty patients had r-EBUS guided placement of a coil-tailed FM before stereotactic radiation therapy. Nodule's median long- and short-axis diameters were $15 \mathrm{~mm}(8-25 \mathrm{~mm})$ and $8 \mathrm{~mm}(5-20 \mathrm{~mm})$, respectively; short diameter of 27 nodules (90\%) was less than $15 \mathrm{~mm}$. All nodules were reached and visualized with r-EBUS, with an ultrasound (US) signal showing a centered or tangential probe in 26 and 4 cases, respectively. No immediate complication was reported. Twenty-three patients had stereotactic radiation therapy within a median time of 29 days (14-126 days). No FM migration occurred between r-EBUS placement and radiotherapy. Pre-treatment planning and 3-month follow-up CT scans showed that all FMs stayed in direct contact with the lesions.

Conclusions: r-EBUS is a safe procedure for the placement of nitinol coil FMs, which have a low migration rate.

Keywords: Bronchoscopy; fiducial marker (FM); stereotactic radiation therapy; peripheral pulmonary nodule; lung cancer; radial endobronchial ultrasonography (r-EBUS)

Submitted May 04, 2019. Accepted for publication Feb 07, 2020.

doi: $10.21037 /$ jtd.2020.02.37

View this article at: http://dx.doi.org/10.21037/jtd.2020.02.37 


\section{Introduction}

Stereotactic radiotherapy constitutes an alternative therapeutic option for peripheral lung cancer when the patient is not suitable for surgery (1). Placement of fiducial gold markers (FMs) into or at the immediate vicinity of the nodule is useful before stereotactic radiotherapy (2). FMs are usually inserted using transthoracic percutaneous technique, which may lead to high rates of pneumothorax $(30 \%)$ and hemoptysis (2\%) (3). Moreover, the migration of FM over the course of treatment is a recognized drawback that may compromise tracking accuracy and could result in inaccurate dosing to the tumor and surrounding tissues (4).

We recently reported that bronchoscopic methods (especially radial endobronchial ultrasonography (r-EBUS) are relevant alternatives for placing FMs into small peripheral nodules $(<20 \mathrm{~mm})$ before stereotactic lung irradiation (5). These bronchoscopic methods minimize the risk of pneumothorax and appear particularly indicated in patients with respiratory insufficiency. However, FMs migrations also occur, leading to additional procedures before stereotactic irradiation.

Gold FM attached to a nitinol pigtail have been recently designed to minimize migration in the lung tissue (Figure 1). The coil had been designed to be placed using electromagnetic navigation, with good results and reported low rate of migration (6).

Our objective was to assess both the feasibility of r-EBUS placement and the migration rate of coil-tailed FMs.

\section{Methods}

\section{Patients}

This single-center retrospective study was conducted from June 2015 to May 2018. We included all of the patients with a pulmonary nodule of $<25 \mathrm{~mm}$ who had FM placement guided by r-EBUS. Nodules were diagnosed or suspected to be a peripheral primary lung cancer, without mediastinal or extrathoracic dissemination. Results from all patients referred during the study period by the radiation therapist or the oncologist for a FM placement are reported in the present work. Whereas this did not represent a selection criterion, a bronchus sign was present on the CT scan in all patients but one. All patients were considered highrisk surgical candidates, with respiratory or cardiovascular comorbidities. Stereotactic radiotherapy FM placement were decided during multidisciplinary thoracic oncology meeting.
The study protocol received the approval of the Institutional Review Board for Non-interventional Research of Rouen University Hospital, France (protocol number E2018-28).

\section{$r$-EBUS procedure}

Before each procedure, the nodule location was mapped using virtual bronchoscopy planner software in order to identify the smallest bronchus leading to the lesion (LungPoint planning Bronchus ${ }^{\circledR}$, USA). The endoscopy was performed under local or general anesthesia using a fiberoptic endoscope with a $4 \mathrm{~mm}$ outer diameter and a $2 \mathrm{~mm}$ working channel (BF-MP60F Olympus ${ }^{\circledR}$, Tokyo, Japan). We used UM-S20-17S ultrasound probe (Olympus ${ }^{\circledR}$, Tokyo, Japan) (Figure 2A), $1.4 \mathrm{~mm}$ in diameter introduced into a guide sheath catheter $1.9 \mathrm{~mm}$ in diameter (K201, Olympus ${ }^{\circledR}$, Tokyo, Japan). A dedicated blocker of the Olympus Kit K201, was placed on the brush in order that the brush get out exactly in same length than the r-EBUS probe.

After the fiberscope had reached the most distal subsegmental bronchus leading to the lesion previously defined in virtual endoscopy, the r-EBUS probe covered with the guide sheath was inserted into the working channel and pushed until a nodule-specific ultrasound image could be obtained. Once the nodule was located via ultrasound (Figure 2B), the probe was retracted and the guide sheath left in place within the working channel, for sampling, before FM placement. No Rapid on Site Examination (ROSE) was performed.

In order to insert the FM with an EBUS guide sheath, we used the following technique: the FM loader tip (SuperLock $^{\mathrm{TM}}$ Nitinol Coil Fiducial Marker; Covidien, Inc., Minneapolis, MN, USA) was inserted into the proximal tip of the bronchial brush sheath after having removed the small metallic wire attached to the brush (Figure $3 A, B, C$, and Video 1). Then, the brush was introduced into the loader allowing the FM to be pushed down to the distal tip of the brush sheath. Finally, the bronchial brush was inserted into the guide sheath and the brush was pushed to the distal tip of the sheath to place the FM into the pulmonary nodule exactly in the same place where was the R-EBUS probe. We did not use fluoroscopy.

After each procedure a chest X-ray was done to ensure the absence of pneumothorax and to visualize the FM.

We assessed the position of the FM and the distance to the nodule on CT scan prior to and 3 months after stereotactic radiotherapy (Figure 4A,B). 


\section{Results}

\section{Patients}

From June 2015 to May 2018, 30 patients had r-EBUS guided placement of a SuperLock ${ }^{\mathrm{TM}}$ Nitinol Coil FM for stereotactic radiation therapy purposes. These procedures

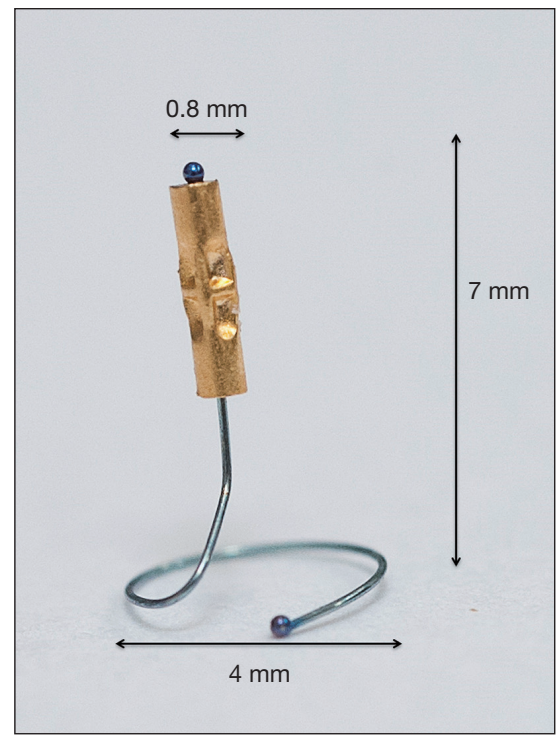

Figure 1 Nitinol Coil tail fiducial marker (Superlock Cobra; Covidien, Inc., Minneapolis, MN, USA). represent $3.5 \%$ of all $\mathrm{r}$-EBUS procedures done in our University Hospital (Figure 5).

Characteristics of patients and nodules are summarized in Table 1. Fourteen patients had a prior major resection, 8 patients had a respiratory contraindication for surgery due to impaired respiratory function, 3 patients had previous radiotherapy, 2 patients were too old (82 and 83 years, respectively) and 2 patients had a cardiovascular contraindication to surgical treatment, and 1 declined the surgery. The median forced expiratory volume in 1 second (FEV1) was $60 \%$ [ $\min$ to max: $30-100 \%$ ]. The median diameter of the nodules was $15 \mathrm{~mm}$ (min to max: $8-25 \mathrm{~mm}$ ) for the long axis and $8 \mathrm{~mm}$ (min to max: $5-20 \mathrm{~mm}$ ) for the short axis; 27 out of 30 nodules $(90 \%)$ exhibited a short diameter of less than $15 \mathrm{~mm}$. A bronchus sign was present on CT in 29 cases (97\%). The median distance was $14 \mathrm{~mm}$ (min to max: 0-53 $\mathrm{mm}$ ) between the nodule and pleura.

\section{Procedure}

The procedure was performed under local anesthesia without sedation in 18 cases $(60 \%)$ and under general anaesthesia in 12 cases (40\%). All nodules were visualized with r-EBUS showing a centered ultrasound (US) signal in 26 and a tangential signal in 4 cases.

All nodules were sampled for histology by r-EBUS during the same procedure as FM placement, including
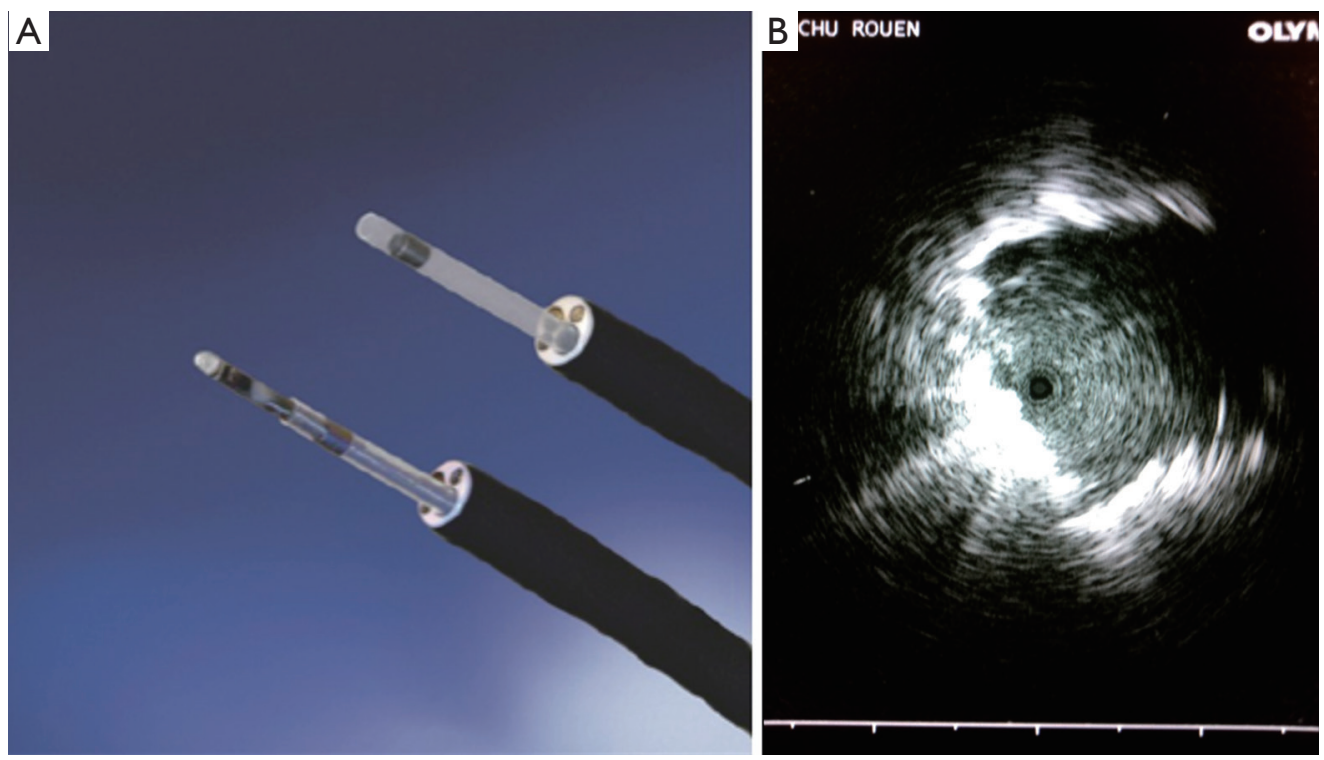

Figure 2 Radial EBUS with guide sheath and ultrasound image. (A) UM-S20-17S radial probe (Olympus®, Tokyo, Japan) introduced into the $1.9 \mathrm{~mm}$-diameter guide sheath; (B) ultrasound tangential image of nodule. EBUS, endobronchial ultrasonography. 

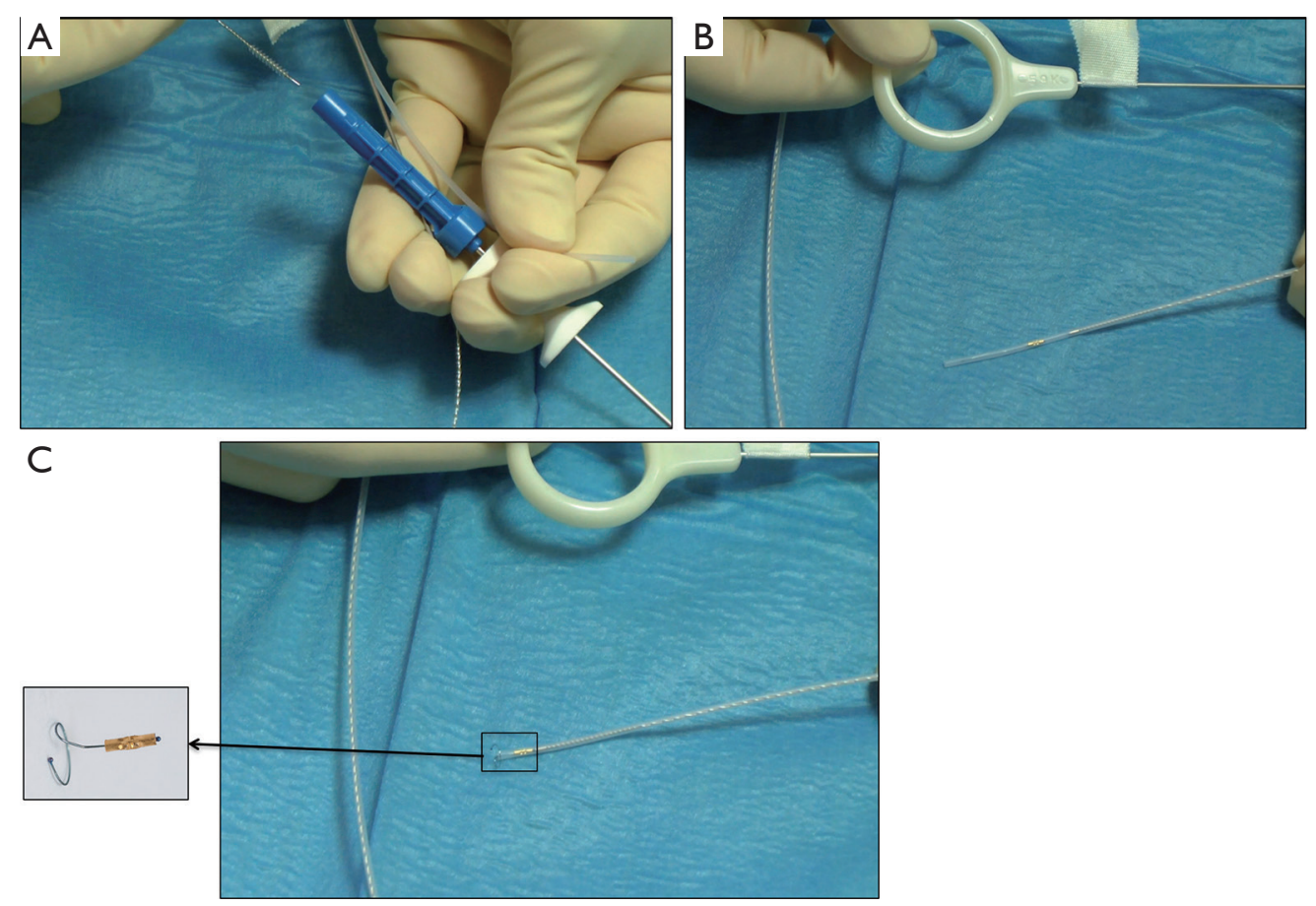

Figure 3 Coil tailed FM placement procedure. (A) The coil tail fiducial marker is inserted into the proximal tip of the bronchial brush sheath after having removed the small metallic wire attached to the brush. (B,C) Then, the brush is reintroduced and the fiducial marker is pushed to the distal tip of the brush. This bronchial brush is inserted into the guide sheath and the brush is pushed to place the FM in the pulmonary nodule.

24 cases for which the histology was not known before the procedure. Histological diagnosis was obtained during the r-EBUS-FM placement procedure in 25 of the 30 patients, leading to a confirmed histological diagnosis of cancer in $83 \%$. Among the 25 patients with a definite diagnosis of cancer confirmed on histology, three had successful surgical resection of the nodule after pulmonary rehabilitation, while 21 benefited from stereotactic radiotherapy and one refused further treatment.

Among the 5 patients without available histology before treatment, one had minimally invasive sublobar surgery after pulmonary rehabilitation, one is still under follow-up without treatment, one received palliative chemotherapy because of metastatic progression, while 2 patients with a nodule that had increased in size and that was hypermetabolic on 5FDG PET CT scan benefited from stereotactic radiotherapy without histological confirmation of cancer (Figure 5).

\section{FM placement and follow-up}

A single FM was inserted per lesion. No complication (pneumothorax or haemorrhage) was reported. For the 23 patients who had stereotactic radiation therapy, there was a median 29 days (min to max: 14-126 days) delay between FM placement and stereotactic radiotherapy. No FM migration was reported between r-EBUS placement and radiotherapy. All lesions subjected to stereotactic radiotherapy were in direct contact with the FM on the pretreatment planning CT and on the 3-month follow-up CT scan after the treatment.

For the 7 patients not treated by radiotherapy, no FM migration was reported: among the 4 patients treated by surgery, the median delay between FM placement and surgery was approximately 85 days (50-161 days), and all the FMs were found within the nodule in the resection specimen on histological analysis. For the 3 patients still on follow-up without treatment or treated by chemotherapy, the FMs were still present and in contact with the lesion on the last CT scan 9, 18 and 28 months after fiducial placement, respectively.

We reviewed all of the CT scan and r-EBUS images to assess the final placement of FM on CT according to the nature (centered or tangential) of the US signal. For the 26 patients who add a centered US image on r-EBUS we 

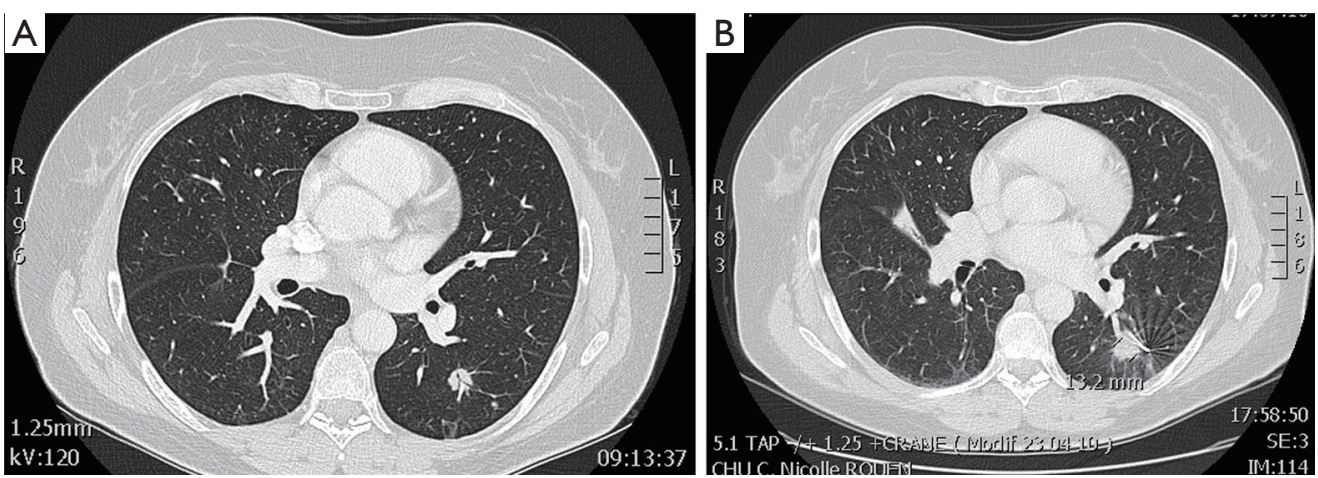

Figure 4 Chest CT scan showing persisting fiducial marker (FM) in a small peripheral nodule after stereotactic radiation. (A) Small peripheral nodule before FM placement. (B) The same nodule 3 months after radiation therapy, with the FM still in place.

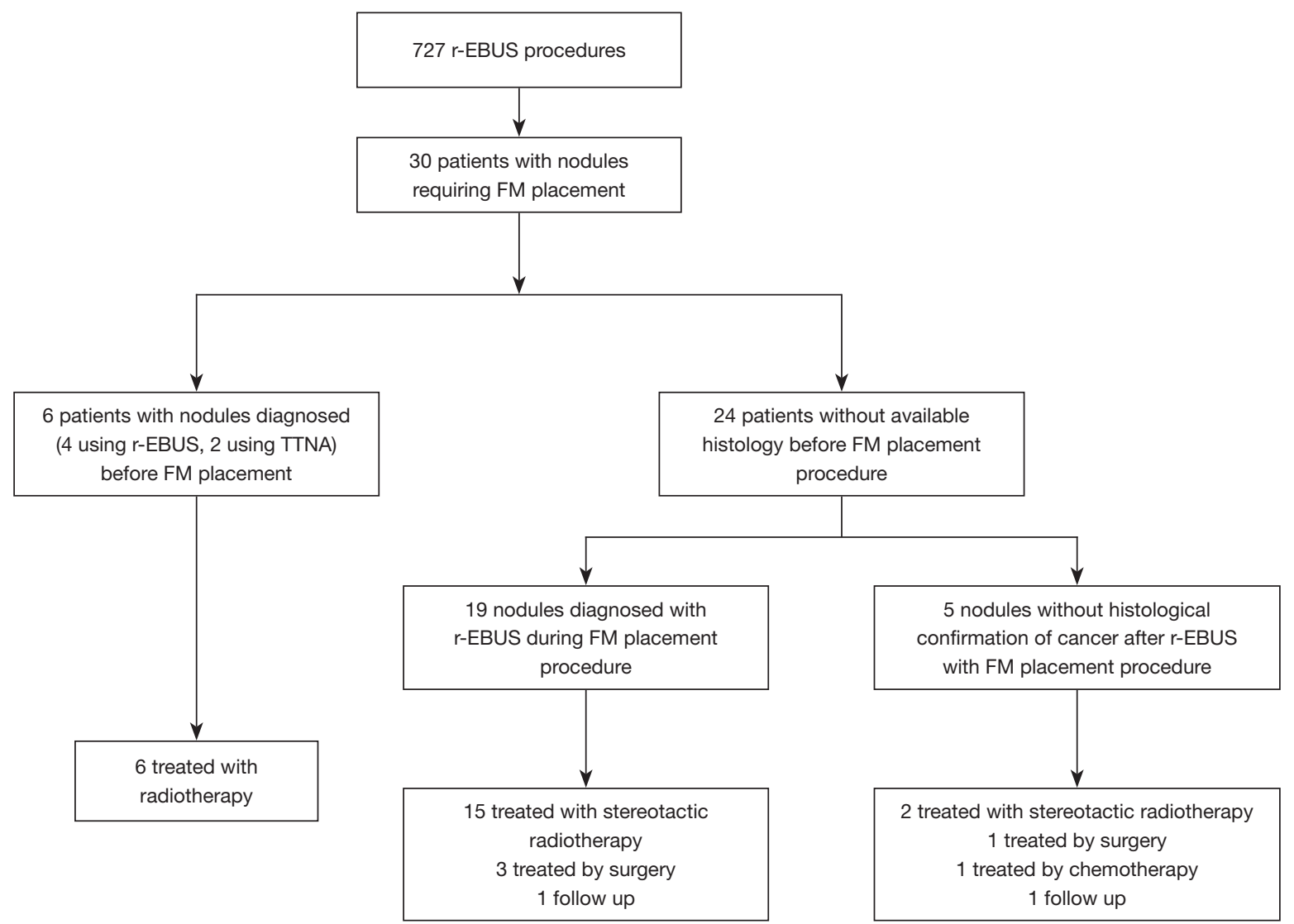

Figure 5 Flowchart. FM, fiducial marker; r-EBUS, radial endobronchial ultrasound; TTNA, trans-thoracic needle aspiration.

found that the FM was placed on the lesion, and for the 4 patients who add a tangential image the FM was placed on the peripheral of the lesion.

Twenty-five patients (95\%) were alive and no recurrence was observed at the study endpoint, after a median 18 months (min to max: 1-37 months) follow-up. Two patients died 24 months after chemotherapy initiation, due to cancer progression. 
Table 1 Patients' and nodule's characteristics

\begin{tabular}{|c|c|}
\hline Characteristics & Values \\
\hline Age (years), mean [min to max] & 68.5 [44-83] \\
\hline Gender ( $\mathrm{n}$, male/female) & $21 / 9$ \\
\hline Previous history of lung cancer & $20(67 \%)$ \\
\hline History of major lung resection & $\begin{array}{l}14 \text { (12 lobectomy, } \\
2 \text { pneumonectomy) }\end{array}$ \\
\hline Previous history of thoracic radiotherapy (n) & 3 \\
\hline \multicolumn{2}{|l|}{ Median nodule diameter $( \pm \mathrm{IQR}), \mathrm{mm}$} \\
\hline Long axis & $15[8-25]$ \\
\hline Short axis & 8 [5-20] \\
\hline $\begin{array}{l}\text { Median ( } \pm \text { IQR) nodule-to-pleura } \\
\text { distance }(\mathrm{mm})\end{array}$ & $14[0-53]$ \\
\hline Bronchus sign (yes/no) & $29 / 1$ \\
\hline \multicolumn{2}{|l|}{ Nodule location } \\
\hline Right upper lobe & 7 \\
\hline Right middle lobe & 4 \\
\hline Right lower lobe & 7 \\
\hline Left upper lobe & 8 \\
\hline Left lower lobe & 4 \\
\hline Tangential/central position of US probe & $4 / 26$ \\
\hline $\begin{array}{l}\text { FM placement under local/general } \\
\text { anesthesia }\end{array}$ & $18 / 12$ \\
\hline
\end{tabular}

FM, fiducial marker; US, ultrasound; M, male; F, female.

\section{Discussion}

Pulmonary tumor motion is a significant problem during stereotactic radiation therapy. FM represent one way to mitigate the effects of motion (4). In this series, we show that r-EBUS for the placement of coil-tailed FM for small pulmonary nodules appears efficient without migration on follow-up.

In a previous study we found that r-EBUS for the placement of gold seed FM in peripheral pulmonary nodules of less than $20 \mathrm{~mm}$ is an easy and safe procedure compared to percutaneous methods, which are associated with high rates of pneumothorax and haemorrhage (5). Compared to this study, nodule and patient characteristics appear similar and tolerance of the procedure was very good, with no complication reported. In both studies, software tracking stereotactic radiation therapy could not be used without gold FM as nodules were very peripheral with a median distance to the pleura of about $14 \mathrm{~mm}$ and of very small size $(90 \%$ exhibited a short diameter of less than $15 \mathrm{~mm})$. Also, the diagnostic accuracy of r-EBUS performed during the same procedure as FM placement was over $80 \%$. In addition, we have shown that FM can be placed without the use of fluoroscopy $(7,8)$.

However, the main difference between our two studies was the absence of migration when using this new endobronchial-dedicated nitinol coil FM, compared to $18 \%$ migration in our previous study. As a comparison, studies using percutaneous methods reported FMs migration rates varying from $9 \%$ to $19 \%(9,10)$.

One of the first publications on bronchoscopic method for FM placement used a transbronchial aspiration needle, with the FM dropping into the airway in $22 \%$ of the cases (11).

Recent studies have evaluated navigational bronchoscopy techniques for FM placement (12-19); six using electromagnetic navigation (EMN) and two using r-EBUS. In these studies, various types of FM were used including "seed" and "coil" markers. Coil markers were used in three studies. They seem to be more reliable than seed markers with a rate of migration of about $1 \%$ to $4 \%(17-19) v s .10 \%$ to $50 \%(12-17)$. However, the coil markers used for these studies were not designed for bronchoscopic methods.

To our knowledge, only one study has evaluated the placement of the SuperLock ${ }^{\mathrm{TM}}$ Nitinol Coil FM (6) during navigational bronchoscopy. This study used EMN in 15 patients with 16 nodules with a mean size of approximately $17 \mathrm{~mm}(8-36 \mathrm{~mm})$. Three to $4 \mathrm{FMs}$ were placed adjacent to the lesion (within $2 \mathrm{~cm}$ ) using fluoroscopy under general anesthesia. The chest CT simulator was performed 40 to 48 hours after the procedure, demonstrating that only $7 \%$ of the FMs had $>5 \mathrm{~mm}$ migration.

In our study we report no migration, even after a long delay between FM placement and CT simulator [29 days (min to max: 14-126 days)]. Compared to EMN method, this may be due to the fact that r-EBUS allows the direct visualisation of the distal nodule during the procedure, therefore ascertaining the disposition of the marker inside a solid structure.

In our study, most of the procedures (62\%) were performed under local anesthesia. However general anesthesia or sedation were employed in the previous SuperLock ${ }^{\mathrm{TM}}$ Nitinol Coil FM perendoscopic study using 
EMN with the Superdimension technique, which may be explained by the fact that EMN requires a larger working channel and a larger fiberscope (6 $\mathrm{mm}$ in general). The use of general anaesthesia or sedation is a risk factor in this population with severe chronic obstructive pulmonary disease (COPD) (20). r-EBUS uses a finer bronchoscope (4 mm) compared to EMN (6 mm), which results in a more distal progression of the endoscope, and a more selective catheterization of the subsegmental bronchus.

EMN using the VERAN system is feasible using the same $4 \mathrm{~mm}$ endoscope we used in the present study. However, to date, there is no publication related to the use of the VERAN system, which is currently not available in Europe, for FM placement.

For these reasons, as r-EBUS appears less expensive than $\mathrm{EMN}$, and requires no specific operating room preparation, specific studies comparing these two techniques for FM placement will be relevant.

Whereas, the use of a single FM allows the same tracking performance and is less expensive, the technique described in the present study could be modified in order to allow the placement of several FMs, by inserting the EBUS scope to a subsegmental bronchus adjacent to the targeted lesion.

Besides its retrospective and single center nature, the principal limitation of our study is the absence of a control group which did not allow any comparison between the efficiency and side effects of stereotactic radiation therapy with or without this coil FM. As nodules from all patients but one presented a bronchus sign on CT scan, results may not be extended to peripheral lung nodules (PLN) without bronchus sign.

Therefore, the migration rate of FM will have to be assessed in cases without bronchus sign. Despite these limitations, in our opinion these findings allow a favourable comparison with previous techniques to guide stereotactic radiation therapy.

\section{Conclusions}

r-EBUS appears safe and easy to perform for nitinol coiltailed FM placement in small peripheral nodules, with a very low rate of secondary migration after long term follow-up.

\section{Acknowledgments}

The authors are grateful to Nikki Sabourin-Gibbs, Rouen University Hospital, for her help in editing the manuscript. Funding: None.

\section{Footnote}

Conflicts of Interest: All authors have completed the ICMJE uniform disclosure form (available at http://dx.doi. org/10.21037/jtd.2020.02.37). SL reports personal fees from Olympus, personal fees from Fujifilm, outside the submitted work; MS reports personal fees from Olympus, outside the submitted work. The other authors have no conflicts of interest to declare.

Ethical Statement: The authors are accountable for all aspects of the work in ensuring that questions related to the accuracy or integrity of any part of the work are appropriately investigated and resolved. The study protocol received the approval of the Institutional Review Board for Non-interventional Research of Rouen University Hospital, France (protocol number E2018-28).

Open Access Statement: This is an Open Access article distributed in accordance with the Creative Commons Attribution-NonCommercial-NoDerivs 4.0 International License (CC BY-NC-ND 4.0), which permits the noncommercial replication and distribution of the article with the strict proviso that no changes or edits are made and the original work is properly cited (including links to both the formal publication through the relevant DOI and the license). See: https://creativecommons.org/licenses/by-nc-nd/4.0/.

\section{References}

1. Timmerman R, Paulus R, Galvin J, et al. Stereotactic body radiation therapy for inoperable early stage lung cancer. JAMA 2010;303:1070-6.

2. Murphy MJ. Fiducial-based targeting accuracy for external-beam radiotherapy. Med Phys 2002;29:334-44.

3. Mendiratta-Lala M, Sheiman R, Brook OR, et al. CTguided core biopsy and percutaneous fiducial seed placement in the lung: can these procedures be combined without an increase in complication rate or decrease in technical success? Eur J Radiol 2014;83:720-5.

4. Loo BW Jr, Kavanagh BD, Meyer JL. Motion management and image guidance for thoracic tumor radiotherapy: clinical treatment programs. Front Radiat Ther Oncol 2011;43:271-91.

5. Lachkar S, Guisier F, Roger M, et al. Assessment of PerEndoscopic Placement of Fiducial Gold Markers for Small Peripheral Lung Nodules $<20 \mathrm{~mm}$ Before Stereotactic Radiation Therapy. Chest 2018;153:387-94. 
6. Rong Y, Bazan JG, Sekhon A, et al. Minimal InterFractional Fiducial Migration during Image-Guided Lung Stereotactic Body Radiotherapy Using SuperLock Nitinol Coil Fiducial Markers. PLoS One 2015;10:e0131945.

7. Herth FJ, Eberhardt R, Becker HD, et al. Endobronchial ultrasound-guided transbronchial lung biopsy in fluoroscopically invisible solitary pulmonary nodules: a prospective trial. Chest 2006;129:147-50.

8. Muhm JR, Miller WE, Fontana RS, et al. Lung cancer detected during a screening program using four-month chest radiographs. Radiology 1983;148:609-15.

9. Bhagat N, Fidelman N, Durack JC, et al. Complications associated with the percutaneous insertion of fiducial markers in the thorax. Cardiovasc Intervent Radiol 2010;33:1186-91.

10. Kothary N, Heit JJ, Louie JD, et al. Safety and efficacy of percutaneous fiducial marker implantation for image-guided radiation therapy. J Vasc Interv Radiol 2009;20:235-9.

11. Reichner CA, Collins BT, Gagnon GJ, et al. The placement of gold fiducials for Cyberknife stereotactic radiosurgery using a modified transbronchial needle aspiration technique. J Bronchol 2005;12:2.

12. Anantham D, Feller-Kopman D, Shanmugham LN, et al. Electromagnetic navigation bronchoscopy-guided fiducial placement for robotic stereotactic radiosurgery of lung tumors: a feasibility study. Chest 2007;132:930-5.

13. Steinfort DP, Siva S, Kron T, et al. Multimodality guidance for accurate bronchoscopic insertion of fiducial markers. J Thorac Oncol 2015;10:324-30.

14. Bolton WD, Richey J, Ben-Or S, et al. Electromagnetic

Cite this article as: Lachkar S, Guisier F, Roger M, Bota S, Lerouge D, Piton N, Thiberville L, Salaün M. A simple endoscopic method with radial endobronchial ultrasonography for low-migration rate coil-tailed fiducial marker placement. J Thorac Dis 2020;12(4):1467-1474. doi: 10.21037/jtd.2020.02.37
Navigational Bronchoscopy: A Safe and Effective Method for Fiducial Marker Placement in Lung Cancer Patients. Am Surg 2015;81:659-62.

15. Hagmeyer L, Priegnitz C, Kocher M, et al. Fiducial marker placement via conventional or electromagnetic navigation bronchoscopy (ENB): an interdisciplinary approach to the curative management of lung cancer. Clin Respir J 2016;10:291-7.

16. Harley DP, Krimsky WS, Sarkar S, et al. Fiducial marker placement using endobronchial ultrasound and navigational bronchoscopy for stereotactic radiosurgery: an alternative strategy. Ann Thorac Surg 2010;89:368-73; discussion 373-4.

17. Minnich DJ, Bryant AS, Wei B, et al. Retention Rate of Electromagnetic Navigation Bronchoscopic Placed Fiducial Markers for Lung Radiosurgery. Ann Thorac Surg 2015;100:1163-5; discussion 1165-6.

18. Nabavizadeh N, Zhang J, Elliott DA, et al. Electromagnetic navigational bronchoscopy-guided fiducial markers for lung stereotactic body radiation therapy: analysis of safety, feasibility, and interfraction stability. J Bronchology Interv Pulmonol 2014;21:123-30.

19. Schroeder C, Hejal R, Linden PA. Coil spring fiducial markers placed safely using navigation bronchoscopy in inoperable patients allows accurate delivery of CyberKnife stereotactic radiosurgery. J Thorac Cardiovasc Surg 2010;140:1137-42.

20. Gunnarsson L, Tokics L, Lundquist H, et al. Chronic obstructive pulmonary disease and anaesthesia: formation of atelectasis and gas exchange impairment. Eur Respir J 1991;4:1106-16. 